\title{
Water for thought: is there a role for aquaporin channels in delirium?
}

\section{Adonis Sfera ${ }^{1 *}$ and Carolina Osorio ${ }^{2}$}

1 Psychiatry, Patton State Hospital, Patton, CA, USA

${ }^{2}$ Kaiser Permanente, Fontana, CA, USA

${ }^{*}$ Correspondence: dr.sfera@gmail.com

Edited by:

Barry Boland, University College Cork, Ireland

Reviewed by:

Barry Boland, University College Cork, Ireland

Shane V. Hegarty, University College Cork, Ireland

Keywords: delirium, aquaporins, neuroinflammation, acetylcholine, aquaporin blockers

C. G. Jung believed that water was a symbol for the unconscious mind, the background from where our conscious thoughts emerge, and also the sea where they melt into the dream-like state of primary processes (1).

Water comprises about $80 \%$ of the brain volume and water homeostasis is inextricably coupled to the CNS function. In this regard, neuroscience describes an inverse relationship between the intensity of the neuropil function and the amount of water it contains. For example, during high neuronal activity (such as information processing), water is transported away from the neuropil, shrinking the extracellular matrix (ECM). Conversely, during times of lower brain activity (such as during sleep), water is shifted back to the neuropil, expanding the $\operatorname{ECM}(2,3)$.

The movement of water in and out of the neuropil occurs with the help of the glymphatic system via special molecular pumps, aquaporin water channels (AQP 4) located in astrocyte end-feet. Water circulation is enabled by the exchange between the cerebrospinal fluid (CSF) and interstitial fluid (ISF). The pressure gradient for this exchange is probably provided by pericytes' contraction and arterial pulsations along with the suction, pump-like action of AQP 4 channels (4-6). This movement of water in and out of the neuropil enables both, clearance of molecular waste and volume transmission (VT) of chemical signals (7). Conversely, delayed water movement (glymphatic stasis) may predispose to the accumulation of misfolded proteins (4) and ultimately to neuroinflammation (8).

The relationship between water and delirium is complex. Both, brain edema and dehydration may predispose to delirium (9). Up-regulation of AQP 4 water channels seems to occur in both situations. In fact, a biphasic up-regulation was described in edema build-up and the resolution phase (10). Interestingly, AQP 4 receptors seem to be the common denominator between the neuropil water movement and neuroinflammation (10). Moreover, animal studies demonstrated that peripheral dehydration triggers central upregulation of AQP 4 receptors (11-13). This in turn causes swelling and priming of astrocytes and microglia, predisposing to neuroinflammation (14).

According to a recent study, two key factors, systemic inflammation and central cholinergic impairment must interact in order to produce delirium (15).

The goal of this article is not to discuss VT or the relationship between AQP 4 channels and inflammation since extensive literature exists on these subjects. Instead, we attempt to answer three questions:

Can an inefficient glymphatic clearance lead to impairment of central cholinergic transmission?

Does glymphatic stasis contribute to microglia and astrocytes' priming, the precursor of neuroinflammation?

Do aquaporin blockers have a place in delirium?

We hypothesize that failure of glymphatic clearance leads to impairment of acetylcholine volume transmission (AChVT), contributing to impaired arousal, attention, memory, and sleep as seen in delirium.
We hypothesize further, that glymphatic failure is pro-inflammatory in nature, leading to up-regulation of AQP 4 channels, which in turn trigger astrocyte swelling and gliosis with the end result being microglial and astrocytic priming.

The above phenomena may reconcile two theories of delirium: central cholinergic deficit and neuroinflammation.

\section{ACETYLCHOLINE AND GLYMPHATIC STASIS}

Central cholinergic deficit is the best established neurotransmitter dysfunction in delirium and its role was known for a long time, however, impaired AChVT was not given much thought in the literature in spite of evidence demonstrating that $86-93 \%$ of cholinergic boutons in the CNS do not make synaptic contact, but release acetylcholine (ACh) directly into the ISF (16).

It is generally accepted that chemical communication between neurons can occur by fast, point to point transmission at the synapse, or by non-synaptic interactions in which neurotransmitters are released from axon terminals (without a conventional synaptic contact) directly into the ISF. In this later case, neurotransmitters travel through the ISF by convection and influence the activity of other neurons through stimulation of extrasynaptically located receptors (17).

Failure of glymphatic clearance may lead to accumulation of molecular debris in the ISF, which in turn may impair the "go-with-the-flow" of AChVT. Since VT represents a large proportion of ACh signaling, it was hypothesized that it may support the sustained and widespread neural 
functions such as cognition, attention, awareness, and sleep $(18,19)$. Since these functions are affected in delirium and since low plasma and CSF levels of ACh have been consistently described in delirious patients (20), it is possible to assume that AChVT may play a central role in the pathophysiology of delirium.

The discovery of the glymphatic clearance helps put AChVT in perspective visà-vis neurodegeneration, sleep, and possibly delirium. The link between AQP 4 and cholinergic neurotransmission was demonstrated by animal studies. For example, AQP 4 and chlorine receptors (ClCN3) up-regulation was documented in studies of transgenic mice with Ach deficit (21).

Another interesting animal study documented co-localization of cholinergic muscarinic receptors (mAChRs) and aquaporin-4 (AQP4) water channels on astrocytic membrane (22). Microglia is known to express nicotinic receptors on its membrane. It is also known that activation of these receptors in vitro attenuates pro-inflammatory responses (23). Since microglia also expresses AQP 4 receptors (24), it is possible, that just like in astrocytes, glymphatic stasis leads to a deficit of AChVT and up-regulation of AQP 4 channels on microglial surface. AQP 4 upregulation (which is pro-inflammatory) may constitute the link between cholinergic deficit and neuroinflammation.

On the other hand, the lack of efficacy of rivastigmine in delirium (25) may not invalidate the cholinergic deficit theory. Instead, it may demonstrate the difficulty in correcting ACh function while AQP 4 receptors are up-regulated. It would be interesting to study the efficacy of rivastigmine used concomitantly with an AQP 4 blocker in delirium.

\section{NEUROINFLAMMATION AND GLYMPHATIC STASIS}

The brain lacks a lymphatic circulation or macrophages. For this reason, neuroinflammation is immunologically different from peripheral inflammation. In this respect "priming," which occurs in the CNS prior to neuroinflammation, can be fathomed as being similar to sensitization. Priming was described in both microglia and astrocytes, cells that also express AQP 4 water channels on their surface. In preclinical animal models, it was demonstrated that in response to the accumulation of abnormally folded proteins in the ISF, astrocytes and microglia react by adopting an activated state, and by releasing molecules that drive their own proliferation. When "primed" these cells are susceptible to a secondary inflammatory stimulus that may arise from surgery or other systemic inflammatory process (8). Interestingly, normal aging was also demonstrated to be a stimulus for microglial "priming" (26).

The process of priming may explain both, the increased incidence and the sudden onset of delirium in medically and surgically ill patients (20).

The accumulation of molecular waste in the ISF also affects neuropil hydration, leading to impairment in neuronal excitability and survival. Genetic studies demonstrate the existence of hydrationsensitive genes in the brain, such as clathrin, that influence neuroexcitability, trigger glial swelling, and result in neuropathology (27).

In a 2013 study, our group created a rat model of ischemia of the nucleus basalis of Meynert and hippocampus. Ischemic changes consisted of swelling of astrocytes, pericyte dysfunction, detachment of astrocytic end-feet from the capillaries with release of glial fibrillary acidic protein (GFAP). AQP4 receptors were not directly assessed, but astrocyte edema (visualized by CD3 staining of GFAP antibodies) provided indirect evidence of AQP4 upregulation (28).

Up-regulation of AQP 4 receptors was described in the pathophysiology of delirium due to liver failure (29). Cultured astrocytes treated with ammonia have been shown to undergo cell swelling with increased expression of AQP4 receptors (30).

AQP4 water receptors up-regulation was demonstrated in traumatic brain injury (TBI) (31), ischemia, epilepsy, multiple sclerosis, HIV encephalitis, and progressive multifocal leukoencephalopathy (PML) (32).

Studies of Alzheimer's disease demonstrated enhanced expression of astrocytic AQP4 receptors compared to age-related controls (33). These changes may be caused by the process of aging, especially since it was documented that in senescence astrocytes up-regulate both AQP 4 and Kir
4.1 potassium receptors (34). Moreover, studies in older mice demonstrate upregulation of these receptors, perhaps in order to maintain homeostasis, integrative ability, and adaptation (34). It is interesting that in diabetes mellitus, type 2 , the brain also presents with astrocytic swelling, probably caused by up-regulation of AQP 4. This response was shown to be more pronounced around zones of infarction and it causes delay in vascular repair in the poststroke period. One study showed that metformin prevented astrocyte swelling and facilitated re-vascularization (35). Neuromyelitis optica (NMO) is an inflammatory demyelinating disease that typically affects optic nerves and spinal cord. Autoantibodies against AQP4 are implied in its etiology of NMO (36).

Conversely, decreased expression of AQP4 receptors on astrocyte membrane was found to be neuroprotective (37). For example, in a mouse model of ischemia using AQP4 null mice, intracranial pressure elevation, blood-brain barrier disruption, inflammation, brain edema, and neuronal apoptosis were shown to be reduced in comparison to AQP4 positive mice (38).

Interestingly, melatonin, and melatonin agonist ramelteon were recently found beneficial in delirium (39). The idea of using melatonin in delirium is not new, given that sleep fragmentation and circadian rhythm changes were described previously. The novelty concerns the mechanism of action of melatonin with its ability to block protein kinase $\mathrm{C}$, which inhibits AQP 4 expression (40). In addition, melatonin was shown to be anti-inflammatory, to inhibit nitric oxide synthase and dopamine release (41).

Additional studies are needed to clarify whether neuropsychiatric conditions associated with up regulation of AQP 4 receptors are more likely to predispose to delirium as opposed to situations in which AQP 4 channels are not up-regulated.

\section{DO AOUAPORIN BLOCKERS HAVE A PLACE IN DELIRIUM?}

Studies of ischemia and inflammation in AQP 4-null mice demonstrated decreased astrocytic swelling, and improved overall outcomes and survival (14). With these findings in mind, it was suggested that pharmacological modulation of AQP4 expression may provide a new addition 
to the medical armamentarium of disorders such as brain edema, glaucoma, tumor growth, $\mathrm{CHF}$, and obesity in which water and solute transport are involved (42). Since AQP 4 up-regulation, astrocytic swelling and microglia/astrocyte priming may occur in delirium as a result of glymphatic stasis, would it be reasonable to expect that AQP 4 blockers might be beneficial in delirium?

A newly discovered arylsulfonamide (Aq B013) is an antagonist of AQP 1 and AQP 4. This is a pharmacologic agent that offers a translational promise in the treatment of conditions manifested by upregulation of aquaporins (43). Could it also be beneficial in delirium?

In an animal study, piroxicam was demonstrated to be a potent AQP4 regulator, rendering neuroprotection in focal cerebral ischemia (44). In NMO, an attempt was made at blocking pathogenic NMO-IgG binding to its target, AQP4 by recombinant monoclonal anti-AQP4 antibodies (45).

\section{CONCLUSION}

Impairment of cognition, attention, awareness, and sleep are encountered both, in delirium and Ach deficit. Because the implementation of these functions requires sustained and widespread neuronal activity, it has been proposed that they may be promoted better by volume, rather than synaptic transmission of Ach, which is best suited for fast and selective signals.

Glymphatic stasis has two possible consequences that may be of interest for delirium: impairment of AchVT and priming of microglia and astrocytes. Both of these phenomena are associated with upregulation of AQP 4 receptors, which may be the common denominator between neuroinflammation and cholinergic deficit. Aquaporin blockers, which were found to be useful in cerebral ischemia and stroke, may also be beneficial in delirium alone or in addition to cholinesterase inhibitors.

\section{REFERENCES}

1. Jung CG. Archetypes of the Collective Unconscious. Collected Works of C. G. Jung. 2nd ed. (Vol. 9). New Jersey: Princeton University Press (1968). 451 p.

2. Dudek FE, Rogawski MA. Regulation of brain water: is there a role for aquaporins in epilepsy? Epilepsy Curr (2005) 5(3):104-6. doi:10.1111/j. 1535-7511.2005.05310.x
3. Xie L, Kang H, Xu Q, Chen MJ, Liao Y, Thiyagarajan $\mathrm{M}$, et al. Sleep drives metabolite clearance from the adult brain. Science (2013) 342:373-7. doi:10.1126/science.1241224

4. Yang L, Kress BT, Weber HJ, Thiyagarajan M, Wang B, Deane R, et al. Evaluating glymphatic pathway function utilizing clinically relevant intrathecal infusion of CSF tracer. J Transl Med (2013) 11(1):107. doi:10.1186/1479-5876-11-107

5. Iliff JJ, Hedok L, Yu M, Feng T, Logan J, Nedergaard $\mathrm{M}$, et al. Brain-wide pathway for waste clearance captured by contrast-enhanced MRI. J Clin Invest (2013) 123:1299-309. doi:10.1172/JCI67677

6. Iliff JJ, Wang M, Liao Y, Plogg BA, Peng W, Gundersen GA, et al. Paravascular pathway facilitates CSF flow through the brain parenchyma and the clearance of interstitial solutes, including amyloid beta. Sci Transl Med (2012) 4:147ral11. doi:10. 1126/scitranslmed.3003748

7. Taber KH, Hurley RA. Volume transmission in the brain: beyond the synapse. J Neuropsychiatry Clin Neurosci (2014) 26:4. doi:10.1176/appi. neuropsych.13110351

8. Perry VH, Holmes C. Microglial priming in neurodegenerative disease. Nat Rev Neurol (2014) 10:217-24. doi:10.1038/nrneurol.2014.38

9. Thomas DR, Cote TR, Lawhorne L, Levenson SA, Rubenstein LZ, Smith DA, et al. Understanding clinical dehydration and its treatment. J Am Med Dir Assoc (2008) 9(5):292-301. doi:10.1016/j. jamda.2008.03.006

10. Tourdias T, Mori N, Dragonu I, Cassagno N, Boiziau C, Aussudre J, et al. Differential aquaporin 4 expression during edema build-up and resolution phases of brain inflammation. J Neuroinflammation (2011) 8:143. doi:10.1186/1742-20948- 143

11. Saito N, Ikegami H, Shimada K. Effect of water deprivation on aquaporin 4 (AQP4) mRNA expression in chickens (Gallus domesticus). Brain Res Mol Brain Res (2005) 141(2):193-7. doi:10. 1016/j.molbrainres.2005.09.005

12. Umenishi F, Verkman AS, Gropper MA. Quantitative analysis of aquaporin mRNA expression in rat tissues by RNase protection assay. DNA Cell Biol (1996) 6:475-80. doi:10.1089/dna.1996.15.475

13. Kolber BJ, Key M, Yanfang C, Morris M, Krane C. Aquaporin 4 mRNA expression in mouse brain is induced in response to dehydration. FASEB (2003) 17:4-5.

14. Fukuda AM, Badaut J. Aquaporin 4: a player in cerebral edema and neuroinflammation. J Neuroinflammation (2012) 9:279. doi:10.1186/17422094-9-279

15. Field RH, Gossen A, Cunningham C. Prior pathology in the basal forebrain cholinergic system predisposes to inflammation-induced working memory deficits: reconciling inflammatory and cholinergic hypotheses of delirium. J Neurosci (2012) 32(18):6288-94. doi:10.1523/JNEUROSCI.467311.2012

16. Lendvai B, Vizi ES. Nonsynaptic chemical transmission through nicotinic receptors. Physiol Rev (2008) 88:333-49. doi:10.1152/physrev.00040. 2006

17. Agnati LF, Zoli M, Strömberg I, Fuxe K. Intercellular communication in the brain: wiring versus volume transmission. Neuroscience
(1995) 69(3):711-26. doi:10.1016/0306-4522(95) 00308-6

18. Hasselmo ME, McGaughy J. High acetylcholine sets circuit dynamics for attention and encoding; low acetylcholine sets dynamics for consolidation. Prog Brain Res (2004) 145:207-34. doi:10.1016/ S0079-6123(03)45015-2

19. Trueta C, De-Miguel FF. Extrasynaptic exocytosis and its mechanisms: a source of molecules mediating volume transmission in the nervous system. Front Physiol (2012) 3:319. doi:10.3389/fphys 2012.00319

20. Maldonado JR. Pathoetiological model of delirium: a comprehensive understanding of the neurobiology of delirium and an evidence-based approach to prevention and treatment. Crit Care Clin (2008) 24(4):789-856. doi:10.1016/j.ccc. 2008.06.004

21. Meshorer E, Inbal E, Biton IE, Ben-Shaul Y, BenAri S, Assaf Y, et al. Chronic cholinergic imbalances promote brain diffusion and transport abnormalities. FASEB J (2005) 19(8):910-22. doi:10.1096/fj. 04-2957com

22. Badauta J, Verbavatz JM, Freund-Merciera MJ, Lasbennesa F. Presence of aquaporin-4 and muscarinic receptors in astrocytes and ependymal cells in rat brain: a clue to a common function? Neurosci Lett (2000) 292(2):75-8.

23. van Gool WA. Brain disorders in critical illness. In: Stevens RD, Sharshar T, Wesley EE, editors. Mechanisms, Diagnosis, and Treatment. Cambridge: Cambridge University Press (2013). 141 p.

24. Tomás-Camardiel T, Venero JL, de Pablos RM, Rite I, Machado A, Cano J. In vivo expression of aquaporin-4 by reactive microglia. J Neurochem (2004) 91(4):891-9. doi:10.1111/j.14714159.2004.02759.x

25. van Eijk MM, Roes KC, Honing ML, Kuiper MA, Karakus A, van der Jagt M, et al. Effect of rivastigmine as an adjunct to usual care with haloperidol on duration of delirium and mortality in critically ill patients: a multicentre, double-blind, placebo-controlled randomised trial. Lancet (2010) 376(9755):1829-37. doi:10. 1016/S0140-6736(10)61855-7

26. Dilger RN, Johnson RW. Aging, microglial cell priming, and the discordant central inflammatory response to signals from the peripheral immune system. J Leukoc Biol (2008) 84(4):932-9. doi:10. 1189/jlb.0208108

27. Tang C, Zelenak C, Völkl J, Eichenmüller M, Regel I, Fröhlich H, et al. Hydration-sensitive gene expression in brain. Cell Physiol Biochem (2011) 27:757-68. doi:10.1159/000330084

28. Marinescu D, Mogoanta L, Sfera A, Gradini R, Osorio C, Preda A. An experimental model of ischemia-induced changes into the glymphatic system of the brain. Rom J Psychopharma (2013) 13(4):243-51.

29. Rao KVR, Norenberg MD. Aquaporin-4 in hepatic encephalopathy. Metab Brain Dis (2007) 22(34):265-75.

30. Rama KVR, Chen M, Simard JM, Norenberg MD. Increased aquaporin-4 expression in ammoniatreated cultured astrocytes. Neuroreport (2003) 14(18):2379-82.

31. Shenaqa M, Kassema H, Penga C, Schaferb S, Ding JY, Fredricksona V, et al. Neuronal damage and 
functional deficits are ameliorated by inhibition of aquaporin and HIF1 $\alpha$ after traumatic brain injury (TBI). J Neurol Sci (2012) 323(1-2):134-40. doi:10.1016/j.jns.2012.08.036

32. Florence CM, Baillie LD, Mulligan SJ. Dynamic volume changes in astrocytes are an intrinsic phenomenon mediated by bicarbonate ion flux. PLoS One (2012) 7(11):e51124. doi:10.1371/journal. pone.0051124

33. Moftakhar P, Lynch MD, Pomakian JL, Vinters HV. Aquaporin expression in the brains of patients with or without cerebral amyloid angiopathy. $J$ Neuropathol Exp Neurol (2010) 69(12):1201-9. doi:10.1097/NEN.0b013e3181fd252c

34. Gupta RK, Kanungo M. Glial molecular alterations with mouse brain development and aging: up-regulation of the Kir4.1 and aquaporin-4. Age (2013) 35:59-67. doi:10.1007/s11357-0119330-5

35. Roshini P, Weiguo L, Zhi Q, Johnson MA, Fagan SC, Ergul A. Vascularization pattern after ischemic stroke is different in control versus diabetic rats: relevance to stroke recovery. Stroke (2013) 44:2875-83. doi:10.1161/STROKEAHA. 113.001660

36. Roemer SF, Parisi JE, Lennon VA, Benarroch EE, Lassmann H, Bruck W, et al. Pattern-specific loss of aquaporin-4 immunoreactivity distinguishes neuromyelitis optica from multiple sclerosis. Brain (2007) 130(5):1194-205. doi:10.1093/ brain/awl371

37. Akdemir G, Ratelade J, Asavapanumas N, Verkman AS. Neuroprotective effect of aquaporin-4 deficiency in a mouse model of severe global cerebral ischemia produced by transient 4 -vessel occlusion. Neurosci Lett (2014). doi:10.1016/j. neulet.2014.03.073

38. Katada R, Akdemir G, Asavapanumas N, Ratelade J, Zhang H, Verkman AS. Greatly improved survival and neuroprotection in aquaporin-4knockout mice following global cerebral ischemia. FASEB J (2014) 28(2):705-14. doi:10.1096/fj.13231274

39. Hatta K, Kishi Y, Wada K, Takeuchi T, Odawara T, Usui C, et al. Preventive effects of ramelteon on delirium a randomized placebo-controlled trial. JAMA Psychiatry (2014) 71(4):397-403. doi:10. 1001/jamapsychiatry.2013.3320

40. Bhattacharyaa P, Kumar A, Paulb PS, Patnaikb R. Melatonin renders neuroprotection by protein kinase $\mathrm{C}$ mediated aquaporin-4 inhibition in animal model of focal cerebral ischemia. Life Sci (2014) 100(2):97-109. doi:10.1016/j.lfs.2014. 01.085

41. Esposito E, Cuzzocrea S. Antiinflammatory activity of melatonin in central nervous system. Curr Neuropharmacol (2010) 8(3):228-42. doi:10.2174/ 157015910792246155

42. Frigeri A, Nicchia GP, Svelto M. Aquaporins as targets for drug discovery. Curr Pharm Des (2007) 13(23):2421-7. doi:10.2174/138161207781368738

43. Yool AJ, Brown EA, Flynn GA. Roles for novel pharmacological blockers of aquaporins in the treatment of brain oedema and cancer. Clin Exp Pharmacol Physiol (2010) 37(4):403-9. doi:10.1111/j. 1440-1681.2009.05244.x
44. Bhattacharya P, Kumar A, Dileep PR, Yavagal DR. Aquaporin-4 inhibition mediates piroxicam-induced neuroprotection against focal cerebral ischemia/reperfusion injury in rodents. PLoS One (2013) 8(9):e73481. doi:10.1371/ journal.pone.0073481

45. Zhang H, Saadoun S, Phuan PW, Lam C, Papadopoulos MC, Bennett JL, et al. Antiaquaporin-4 monoclonal antibody blocker therapy for neuromyelitis optica. Ann Neurol (2012) 71(3):314-22. doi:10.1002/ana.22657

Conflict of Interest Statement: The authors declare that the research was conducted in the absence of any commercial or financial relationships that could be construed as a potential conflict of interest.

Received: 13 April 2014; accepted: 12 May 2014; published online: 26 May 2014.

Citation: Sfera A and Osorio C (2014) Water for thought: is there a role for aquaporin channels in delirium? Front. Psychiatry 5:57. doi: 10.3389/fpsyt.2014.00057

This article was submitted to Neurodegeneration, a section of the journal Frontiers in Psychiatry.

Copyright (c) 2014 Sfera and Osorio. This is an openaccess article distributed under the terms of the Creative Commons Attribution License (CC BY). The use, distribution or reproduction in other forums is permitted, provided the original author(s) or licensor are credited and that the original publication in this journal is cited, in accordance with accepted academic practice. No use, distribution or reproduction is permitted which does not comply with these terms. 